\title{
CLASSIFICAÇÃO SUPERVISIONADA DE COPAS DE ÁRVORES EM IMAGEM DE ALTA RESOLUÇÃO ESPACIAL
}

\author{
SUPERVISED CLASSIFICATION OF TREE CROWS IN HIGH SPATIAL RESOLUTION IMAGE
}
Franciel Eduardo Rex 1 , Pâmela Suélen Käfer ${ }^{2}$, Fábio Marcelo Breunig ${ }^{3}$, Renato Beppler Spohr Renato Souza Santos ${ }^{5}$

${ }^{1}$ Universidade Federal do Paraná, Curitiba, Paraná, Brasil-francielrexx@gmail.com

${ }^{2}$ Universidade Federal do Rio Grande do Sul, Porto Alegre, Rio Grande do Sul, Brasil pamelaskafer@gmail.com

3, 4, 5 Universidade Federal de Santa Maria, Frederico Westphalen, Rio Grande do Sul, Brasil breunig@ufsm.br, renatospohr@smail.ufsm.br, renato.souza.santoszf@gmail.com

\section{RESUMO}

\begin{abstract}
O presente estudo teve como objetivo avaliar o potencial de uso de imagens de alta resolução espacial para a extração da área de copa de árvores em floresta nativa utilizando classificação supervisionada. Uma imagem do sensor multiespectral a bordo do satélite Worldview-2 foi adquirida, na qual foram testados três algoritmos de classificação digital de imagens. Com os resultados das classificações, foram delimitadas 51 copas de árvores, que foram localizadas em campo para a coleta de informações como diâmetro na altura do peito (DAP), altura estimada, diâmetro de base, identificação, e medição de 8 raios de copa para formar a área de copa (AC). O algoritmo SVM apresentou o melhor resultado dentre os métodos de classificação testados. Foi encontrado um $R^{2}$ de 0,57 entre $A C$ em campo e o DAP. A relação $A C / D A P$ indica que houve um aumento da área de copa à medida que aumentou o DAP. O $R^{2}$ entre a $A C$ obtida por satélite e o DAP foi de 0,55. A obtenção do parâmetro AC via classificação supervisionada pode servir de base para delimitação de copas, porém, deve-se ter cuidado com este processo para não superestimar as áreas, devido à complexidade do dossel de uma floresta nativa.
\end{abstract}

PALAVRAS-CHAVE: Área de copa, Espécies Florestais, Floresta nativa, Sensoriamento remoto multiespectral.

\section{ABSTRACT}

This paper aimed to evaluate the potential of high spatial resolution images for extraction of the crown area of trees in native forest using supervised classification. Three digital image classification algorithms were tested in an image acquired by the multispectral sensor on the Worldview-2 satellite. Fifty-one crowns were delineated using the classification results, those trees were located in the field to collect information such as diameter at breast height (DAP), estimated height, base diameter, identification, and measurement of eight rays from each crown to form the crowns area (AC). The SVM algorithm presented the best result among the classification methods tested. An $R^{2}$ of 0.57 was found between the crown area $(A C)$ in the field and the DAP. The AC/DAP ratio indicates that there was an increase in crown area as DAP increased. The $R^{2}$ between satellite AC and DAP was 0.55. The obtaining of the AC parameter via supervised classification may be used for crowns delimitation, but it should be cautiously employed in order to avoid overestimating the areas due to the complexity of the native forest crown.

KEYWORDS: Crow area, Forest species, Native forest, Multispectral remote sensing. 


\section{INTRODUÇÃO}

A estimativa de parâmetros florestais sempre foi alvo de estudos de novas técnicas que buscam facilitar a obtenção dos dados, o gerenciamento dos recursos florestais e diminuir os altos custos com inventários (SANTOS et al., 2017). Dado que os métodos tradicionais de coleta de dados são trabalhosos, financeiramente onerosos e, resultam em uma baixa cobertura de amostragem e frequência.

Devido à complexidade das informações para se otimizar o processo de tomada de decisão é comum observar esforços na busca de alternativas (CANAVESI et al., 2010). Nesse contexto, o sensoriamento remoto é uma das mais bem-sucedidas tecnologias de coleta automática de dados para o levantamento e monitoramento dos recursos terrestres, além de ser uma alternativa potencial e de baixo custo no levantamento de campo (MENESES, 2012). O uso de ferramentas de sensoriamento remoto tem se constituído de grande importância nos estudos ambientais, suas imagens polissêmicas e recursos permitem realizar levantamentos periódicos, o que torna possível a aplicação em diferentes temáticas (KALAF et al., 2013).

Utilizando classificação digital, o mapeamento é feito por meio do reconhecimento de padrões e de objetos homogêneos, representados em um conjunto de pixels. $\mathrm{O}$ algoritmo responsável pela classificação digital é denominado "classificador", e a classificação pode ser feita pixel a pixel ou por regiões/objetos (JENSEN, 2011). Para o estudo em nível de copa, ou seja, em nível de indivíduo, imagens obtidas por alguns sensores multiespectrais, possuem resolução espacial com suficiência. Assim, com uma resolução espacial de 0,5 m (pós fusão), o satélite Worldview-2 é uma alternativa viável para mensurar o tamanho das copas de árvores. A partir dessa informação, pode-se derivar outras informações, como o diâmetro na altura do peito, por exemplo (DIGITAL GLOBE, 2010).

Alguns autores em seus estudos já apresentam técnicas que têm sido disseminadas para a mensuração de parâmetros biométricos, a fim de viabilizar o processo de obtenção de dados. Gomes et al. (2008) apresentam métodos de segmentação multi-resolução e classificação orientada a objetos para a identificação de duas espécies de árvores em uma área de Cerradão, enquanto Lima Neto et al. (2012) utilizam fotografias aéreas para mensurar copas de árvores em ambiente urbano. Entretanto, percebe-se que apesar dos estudos e técnicas disponíveis para mensuração de copas de árvores, são poucos os trabalhos que apresentam uma abordagem metodológica de obtenção de parâmetros biométricos em uma floresta nativa, com abordagem pixel a pixel.

Nesse sentido, o objetivo deste trabalho foi testar métodos de classificação supervisionada para a mensuração de copas de árvores em fragmentos de Floresta Estacional Decidual no Norte do Rio Grande do Sul, bem como avaliar o potencial do uso de imagens de alta resolução espacial para obter parâmetros da área de copa.

\section{MATERIAL E MÉTODOS}

\section{Área de estudo}

Este trabalho foi desenvolvido na Floresta Estacional Decidual localizada no município de Frederico Westphalen, nas dependências da Universidade Federal de Santa Maria campus Frederico Westphalen - RS, em uma área de aproximadamente 55 ha dividida em dois fragmentos (Figura 1). De acordo com a classificação de Köppen e Geiger, o clima da região é do tipo Subtropical Temperado úmido (Cfa) (KOTTEK et al., 2006), com temperatura média anual de $18,3^{\circ} \mathrm{C}$ e precipitação média anual de $1.522 \mathrm{~mm}$ (CEMETRS, 2015).

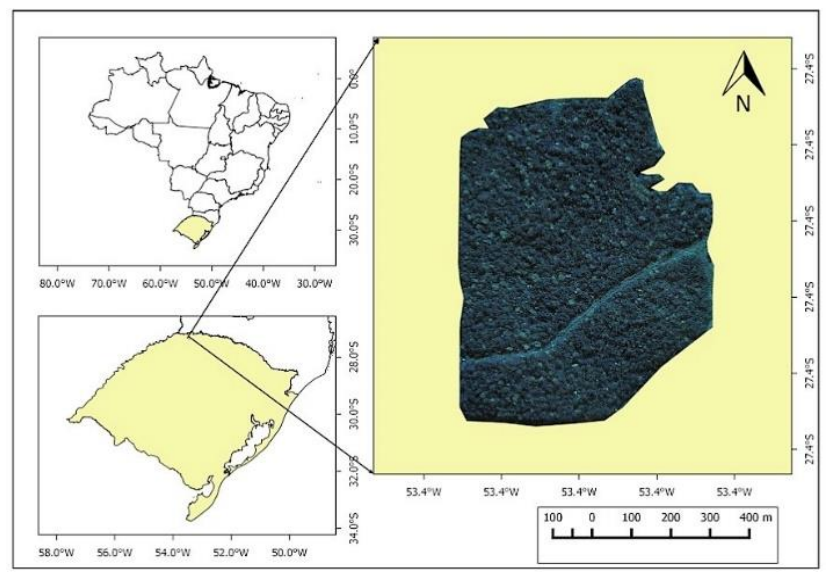

Figura 1. Localização da área de estudo no noroeste do estado do Rio Grande do Sul.

De acordo com Cantarelli et al. (2015), a área de estudo está inserida no bioma Mata Atlântica, onde predominam as formações do tipo Floresta Estacional Decidual. Os autores relatam que esse ambiente é constituído por floresta nativa de aproximadamente 60 anos de idade com relatos de supressão de árvores, presença de clareiras e intervenções antrópicas na área.

\section{MATERIAL E MÉTODOS}

Foi utilizada uma imagem adquirida pelo sensor 
multiespectral a bordo do satélite Worldview-2 em 26/07/2013, com resolução espacial de 0,46 m na banda pancromática e 1,84 $\mathrm{m}$ nas bandas multiespectrais. As imagens multiespectrais foram reamostradas (fusão) para $0,50 \mathrm{~m}$. As imagens possuem resolução radiométrica de 11 bits (DIGITAL GLOBE., 2010). A cena foi adquirida com a ortorrectificação aplicada (base altimétrica do Shuttle Radar Topography Mission - SRTM) e foi realizada a correção atmosférica por meio do Fast Line-of-sight Atmospheric Analysis of Spectral Hypercubes (FLAASH), implementado no aplicativo Envi 5.1 (EXELIS VIS, 2014).

Para o desenvolvimento deste estudo, foram geradas classificações supervisionadas do dossel florestal e delimitações vetoriais de copas das árvores. Os resultados das classificações, foram utilizados para aprimorar a delimitação das copas das árvores dominantes. Foi utilizado o aplicativo do GPS para o pós-processamento dos pontos coletados em campo, com base em um equipamento de posicionamento diferencial e com o uso de uma haste telescópica de $8 \mathrm{~m}$, para elevar a posição da antena, incrementando a qualidade do sinal recebido e aumentando a acurácia posicional.

As classificações supervisionadas utilizadas neste trabalho foram realizadas com o aplicativo Envi 5.1, sendo: Máxima Verossimilhança (MaxVer), que é a técnica de classificação supervisionada mais popular para tratamento de dados satélites; algoritmo Support Vector Machine (SVM), que consiste em treinar um classificador de forma que esse aprenda um mapeamento por meio de exemplos e seja capaz de classificar um exemplo ainda não visto com mesma distribuição probabilística (NASCIMENTO et al., 2009); e o classificador de distância de Mahalanobis que tem por base o classificador de mínima distância. As classes de cobertura foram dosséis e sombra (apenas o talhão de floresta foi utilizado como input nas classificações). Para a avaliação das classificações foi utilizado o índice Kappa (k), que mede o grau de concordância da classificação digital com a referência (PONZONI \& REZENDE, 2002) e o índice de exatidão global (G).

Com base na melhor classificação, foram delimitadas as copas de 51 árvores para inspeção em campo. Para tal, foram coletadas as coordenadas centrais de cada copa (na imagem Worldview-2), em que essas foram transferidas para os equipamentos de GPS. Com o auxílio desses equipamentos, os indivíduos foram encontrados em campo. Dessa forma, foram coletados os dados das espécies para posteriormente comparar com os dados obtidos em nível orbital.

Em campo, foram levantados os seguintes parâmetros das árvores: área de copa, diâmetro a altura do peito (1,3 m - DAP), altura estimada e identificação das espécies. A metodologia utilizada neste trabalho para obtenção da área de projeção de copa partiu da medição de oito raios, os quais foram medidos em ângulo fixo com o auxílio da bússola a partir da posição Norte $(\mathrm{N})$ e nas demais posições cardeais e colaterais, sendo elas: N, NO, O, SO, S, SE, L, NE. A medida da copa foi obtida a partir da porção central dos caules dos indivíduos até o final da extensão da copa. A área da copa foi calculada pela Equação 1:

$$
A C=\left(\pi *(r c 8)^{2}\right) / 4
$$

Em que: $A C=$ área da copa $\left(\mathrm{m}^{2}\right) ;$ e $r c=$ média dos oito raios de copa $(\mathrm{m})$.

Os materiais utilizados no trabalho de campo foram: trenas métricas para medição dos raios e circunferência; um par de GPS modelo Garmin, para a localização das árvores; um GPS diferencial; uma haste telescópica de $8 \mathrm{~m}$; além de materiais como facão, embalagens para amostras de ramos, bússola e câmera fotográfica para os registros complementares.

\section{Análise de dados}

Inicialmente, foram avaliadas as acurácias das classificações supervisionadas e, em seguida, foi selecionada a que apresentou a melhor acurácia para orientar a seleção das copas dominantes. Com base nesse resultado, foram identificadas 51 copas de árvores.

A avaliação da acurácia das classificações foi determinada a partir do índice Kappa e da exatidão global; esses métodos foram calculados por meio das matrizes de confusão geradas pelos classificadores. O índice Kappa ( $k$ ) foi calculado a partir da Equação 2, conforme descrito por (PONZONI \& REZENDE, 2002):

$$
k=N \Sigma x i i-\sum x i+. x+i / N 2-\sum x i+. x+i
$$

Em que: $N=$ número total de observações; $x i i=$ elementos da diagonal principal da matriz; e $x i+$ e $x+i=$ somatório dos elementos da linha e coluna, respectivamente.

O valor de $k$ expressa a concordância entre a classificação dos dados contidos nos produtos de sensoriamento remoto considerados e a realidade de campo (verdade terrestre), mostrando a qualidade da classificação.

O índice de exatidão global, conforme descrito por Ponzoni \& Rezende (2002), é apresentado pela Equação 3: 


$$
G=\Sigma n i i / n
$$

Em que: $G$ = exatidão global; nii = número de elementos da diagonal da matriz de confusão; e $n=$ número total de observações.

Foi utilizada a correlação de Pearson (GARSOM, 2009) para avaliar o grau de correlação das variáveis DAP e altura total com a $A C$, sendo aplicada para as espécies dominantes mais frequentes no fragmento florestal. Por fim, os resultados de $A C$ medidos em campo e obtidos pela imagem foram analisados por meio do coeficiente de determinação $\left(R^{2}\right)$.

\section{RESULTADOS E DISCUSSÃO}

\section{Classificações e inventário de campo}

Foram aplicadas três classificações para discriminar os dosséis florestais iluminados de áreas com sombra (Figura 2). Os três métodos retornaram bons resultados (Tabela 1), contudo, o SVM mostrou-se mais exato. Nitidamente, percebe-se que o algoritmo distância de Mahalanobis gerou uma cobertura de sombra maior que os demais classificadores. Os resultados das classificações supervisionadas serviram como base para a mensuração das copas para a etapa de campo, necessitando, desta forma, identificar o método mais preciso.

Tabela 1. Índice kappa e precisão geral por método de classificação da imagem Worldview-2.

\begin{tabular}{ccc}
\hline Métodos de classificação & Índice Kappa & Precisão geral \\
\hline SVM & 0,9798 & $98,99 \%$ \\
Máxima Verossimilhança & 0,9707 & $98,53 \%$ \\
Distância Mahalanobis & 0,9324 & $96,62 \%$ \\
\hline
\end{tabular}

Os baixos valores encontrados de confusão entre as classes podem ser explicados pela ampla diferença espectral entre elas e pelo pequeno número das mesmas (dosséis e sombra). Amaral et al. (2009), ao avaliarem e compararem métodos de classificação de imagens para o mapeamento de estádios de sucessão florestal, encontraram resultados com baixo nível de exatidão, o que pode ser explicado pela semelhança espectral existente entre os alvos (estádios inicial, médio e avançado de sucessão florestal), pequena variação entre os valores numéricos dos pixels, existência de sobreposição entre classes e pela baixa resolução espectral dos sensores utilizados.

Ferreira et al. (2016), ao utilizarem diversas abordagens de classificadores na delimitação de copas de espécies florestais, verificaram que o procedimento de classificação orientada a objeto elevou a exatidão média para todos os métodos testados. Esse aumento da exatidão média em classificações orientadas ao objeto tem relação com o fato desta classificação levar em consideração o contexto de inserção do pixel, além de seu valor numérico. Os autores ainda comentam que na prática, é muito difícil realizar o censo de todas as espécies que ocorrem na área, rotular todos os pixels da imagem a certo número de espécies pode implicar numa generalização equivocada. Por esse motivo, opta-se por classificar apenas os pixels com alta probabilidade de pertencer a uma determinada classe.

Apesar das classificações supervisionadas se apresentarem visualmente muito semelhantes, o classificador SVM foi eleito para dar início ao processo de delimitação de copas na imagem, o resultado deste classificador é expresso na Figura $2 b$. Com base nessa classificação, foi definido o processo de identificação de copas manual (utilizando a classificação como base). Após a definição das 51 copas, a partir da imagem Worldview-2, procedeu-se a campanha de campo para as medidas in situ (medidas do fuste e da copa).

No total, foram identificadas 17 espécies, sendo que 13 indivíduos não foram identificados devido à dificuldade de se observar as copas quando muito altas, e/ou por causa do entrelaçamento das copas vizinhas ou presença de lianas e cipós.

Trepadeiras lenhosas como cipós (lianas) podem atingir o topo do dossel da floresta e se desenvolver muito, entrelaçando-se em várias árvores (PUTZ, 1984). Nessa perspectiva, percebeu-se que a dificuldade na identificação de alguns indivíduos está relacionada a presença de lianas que geralmente na floresta acabam competindo com as árvores, além de interferir na sua simetria de crescimento e taxa de mortalidade. Em nível de campo por vezes foi encontrado o entrelaçamento entre copas de indivíduos vizinhos. Assim, grandes erros podem ser cometidos se apenas as imagens de satélite forem utilizadas para a delimitação de copas. 


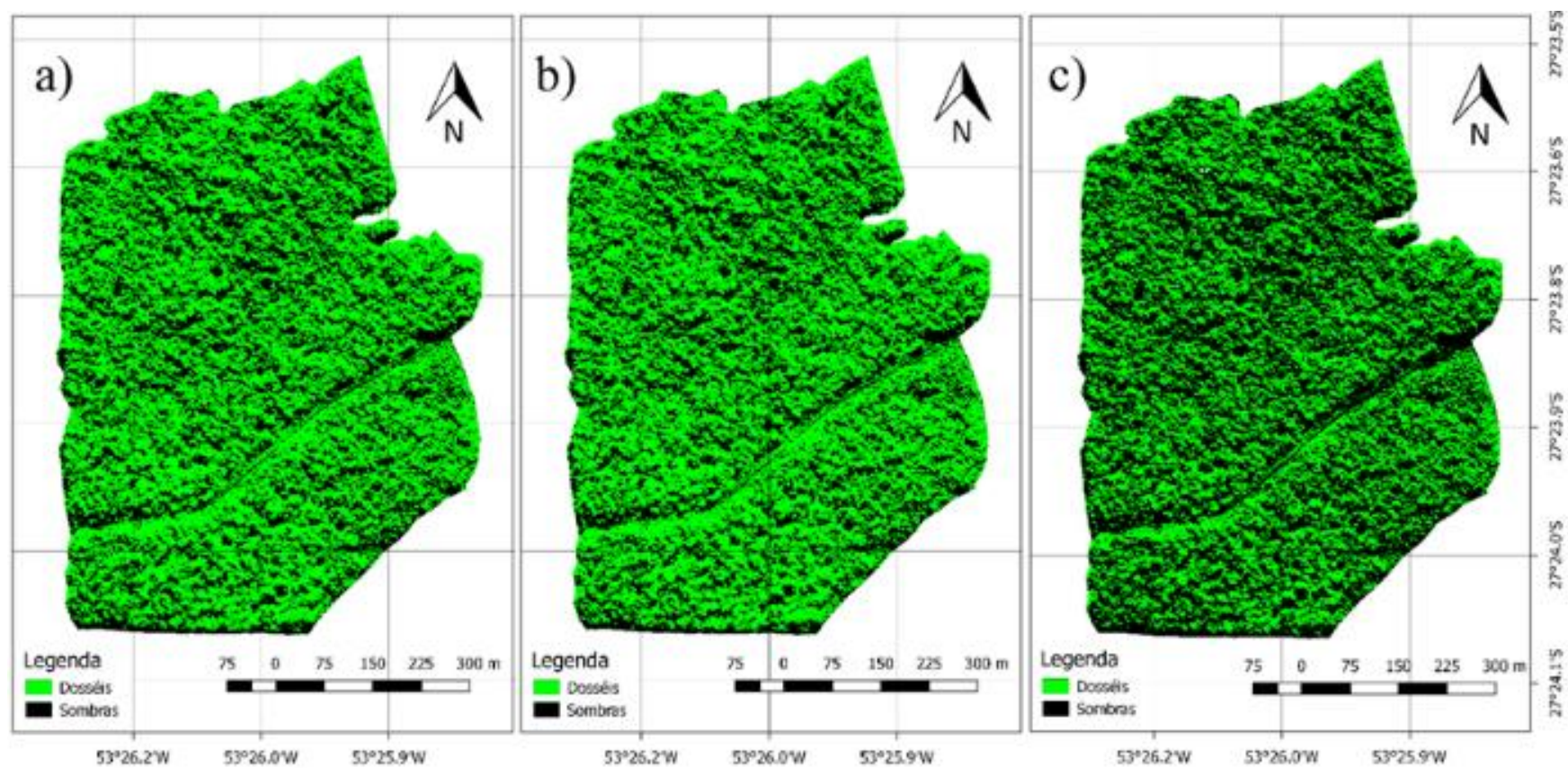

Figura 2. Resultado dos diferentes métodos de classificação para a imagem Worldview-2 de julho de 2013 para identificar copas: a) MaxVer; b) SVM e c) Distância de Mahalanobis.

Na Tabela 2, é observada a relação do DAP em função da $A C$ obtida em campo. De forma geral, as espécies apresentaram uma relação direta do DAP em função da $A C$. Os resultados observados neste trabalho indicaram, de forma geral, que a $A C$ é maior à medida que aumenta o $D A P$. A relação encontrada neste trabalho corrobora com os resultados encontrados por Sanquetta et al. (2014), que encontraram essa relação de aumento do DC à medida que aumenta o DAP em indivíduos de acácia-negra, apesar desta espécie ter uma relação logarítmica, e não uma relação linear como observado neste trabalho.

Observam-se algumas exceções, como o caso da espécie $C$. americana que apresentou a segunda menor média de área de copa e um diâmetro consideravelmente elevado em relação às demais espécies. Esse resultado pode ter sido influenciado pelo número de indivíduos que foi encontrado em campo; outras espécies como a $P$. tetraphylla e o $C$. leiostachya também apresentaram um padrão contrário, sendo que essas também apresentaram apenas um indivíduo.

A baixa representatividade de algumas espécies tornou difícil inferir sobre a probabilidade de determinados acontecimentos. Soares et al. (2006) afirmam que existem sempre erros associados ao ato de medir um objeto. Em um inventário florestal, são realizadas diversas medições das árvores e estas medições podem estar, em maior ou menor grau, afetadas por erros (LIMA NETO et al., 2012).
Dessa forma, ressalta-se que para confirmar esse padrão contrário da relação $D A P / A C$ novos estudos devem ser realizados com essas espécies.

Tabela 2. Valores médios de $D A P, A C$ e $A C$ I lárea de copa referente a imagem) separados por espécie.

\begin{tabular}{cccc}
\hline Nome científico & DAP & AC & AC_I \\
\hline H. Balansae & 50,18 & 16,53 & 14,48 \\
P. Rigida & 87,53 & 30,85 & 29,17 \\
M. Elaeagnoides & 35,01 & 12,32 & 11,07 \\
N. Megapotamica & 46,90 & 12,36 & 12,40 \\
H. Apiculata & 40,74 & 21,27 & 21,69 \\
C. Canjerana & 53,79 & 24,59 & 19,42 \\
C. Fissilis & 53,22 & 27,50 & 33,98 \\
A. Leiocarpa & 67,58 & 27,29 & 24,88 \\
C. Americana & 57,29 & 9,54 & 9,254 \\
A. Parviflorum & 28,96 & 12,09 & 10,75 \\
C. Trichotoma. & 45,91 & 16,51 & 18,78 \\
P. Tetraphylla & 42,65 & 8,03 & 8,69 \\
C. Leiostachya & 42,33 & 12,56 & 12,88 \\
T. Catigua & 36,28 & 11,41 & 11,24 \\
D. Ecastophyllum & 31,19 & 13,92 & 13,89 \\
H. Brasiliensis & 74,40 & 28,13 & 27,44 \\
A. Triplinervia & 80,53 & 31,77 & 27,77 \\
Ni & 48,33 & 19,01 & 18,74 \\
\hline
\end{tabular}


A partir da mensuração das áreas de copa em nível de campo, foram calculadas as áreas e comparadas com as áreas de copa delimitadas a partir da imagem. As áreas de copas que foram delimitadas inicialmente na imagem apresentaram-se maiores que as áreas de copa obtidas em campo. O fato de apresentar uma área maior na imagem de satélite também foi observado por Lima Neto et al. (2012) ao utilizarem fotografias aéreas para mensurar a área de copa das árvores de ruas de Curitiba-PR.

Lima Neto et al. (2012) encontraram um aumento das áreas com o uso de SIG em seis das 11 ruas que estavam sendo avaliadas em comparação aos métodos tradicionais de levantamento de campo. Nesse sentido, pode-se atribuir o aumento elevado das áreas que foram inicialmente delimitadas ao fato de que em uma floresta nativa há muito mais complexidade na delimitação de copas individuais.

Outra hipótese refere-se a defasagem temporal entre a data de aquisição da imagem e o trabalho de campo. A imagem utilizada neste estudo foi adquirida em 2013 e a coleta dos dados foi realizada em 2016, nesse intervalo de tempo muitos distúrbios podem ter ocorrido na floresta, o que pode ter ocasionado mudanças nas áreas de copas coletadas em nível de campo. Além disso, a presença do sombreamento mútuo pode dificultar o delineamento das áreas de copa nas imagens (LIMA NETO et al., 2012). Atualmente, um dos principais parâmetros que devem ser fornecidos junto aos mapas é a avaliação do erro envolvido no produto (POWELL et al., 2004). A Figura 3 ilustra a relação do DAP/AC obtida em nível de campo e por meio da imagem Worldview-2 de todas as espécies em estudo.

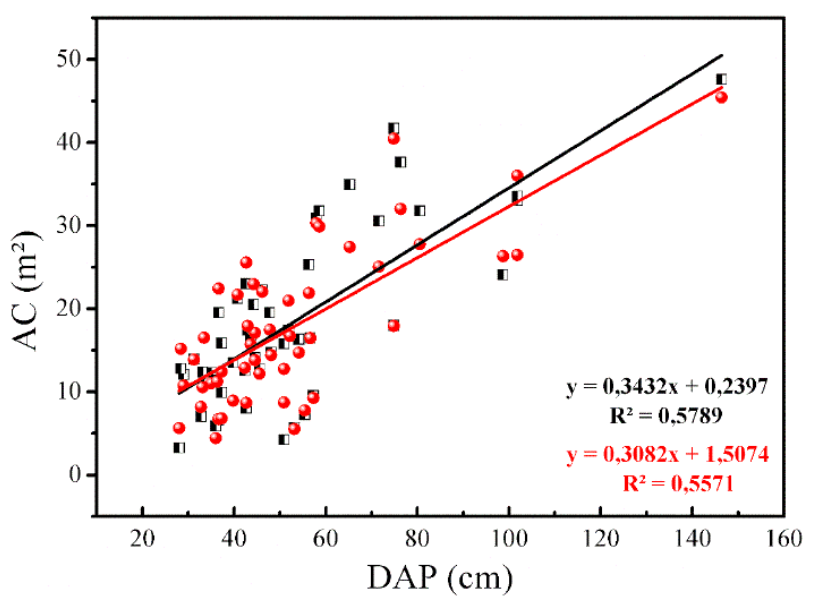

Figura 3. Relação do diâmetro à altura do peito com a área de copa das espécies em estudo, obtida em nível de campo (cor preta) e por meio da imagem Worldview-2 (cor vermelha).

As relações do $D A P / A C$ para os dados deste trabalho foram ajustadas por meio de uma reta linear (Figura 3). Os valores encontrados de $R^{2}$ neste estudo foram de 0,5789 e 0,5571, em nível de campo e na imagem Worldview-2, respectivamente. Observou-se que há uma proporcionalidade entre as maiores áreas de copa com os maiores diâmetros, e vice-versa. Também é possível perceber que alguns indivíduos se revelaram dispersos da média, fato este que inferiu em um valor de $R^{2}$ menor. Há uma elevada concentração de indivíduos nos valores menores de $D A P$ e, além disso, há uma tendência à forma de J-invertido, com maior concentração de árvores nas classes inferiores de diâmetro.

Segundo Odum (2001). a forma de J-invertido é típica de florestas naturais. em que há um grande número de árvores que povoam as classes de menor diâmetro, seguindo uma diminuição exponencial do número de árvores com diâmetros maiores. Callegaro et al. (2012) encontraram esse padrão de distribuição e a tendência à forma de J-invertido ao estudarem a estrutura do componente arbóreo em uma floresta estacional decidual ripária em Jaguari, RS.

Esses resultados indicam que esse padrão pode ter relação com a capacidade de uma floresta em se renovar. Nascimento et al. (2004) citam que a distribuição diamétrica em "J" invertido indica que a floresta tem capacidade de autorregeneração e de manutenção dos níveis atuais de densidade. A relação $D A P / A C$ obtida pela imagem de satélite mostra que o resultado é próximo com o obtido em nível de campo e dada a precisão necessária, pode substituir parcialmente ou complementar as atividades de campo.

Outra forma utilizada para avaliar a relação da $A C$ com as variáveis de fácil obtenção (DAP e altura estimada), foi por meio do coeficiente de correlação de Pearson. Foi identificado que a área de copa de todas as espécies dominantes tem correlação moderada a muito forte com o DAP. Já a correlação entre a área de copa e a altura estimada dos indivíduos se apresentou positivo-fraca e negativo-fraca, com exceção da C. canjerana, desta forma, indicando que para essas espécies dominantes, a altura estimada tem pouca relação com o crescimento da área de copa.

Por fim, e ainda conforme a Figura 3, foi observada a relação entre as copas obtidas na imagem e as obtidas no campo, relacionando todas as árvores e os DAPs versus as copas da imagem. Essa relação demonstrou que o parâmetro área de copa obtido pela imagem Worldview-2 é, de modo geral, bem próximo ao resultado encontrado em campo. Nesse sentido, as áreas obtidas por este sensor podem ser utilizadas para predição de estimativas de 
variáveis dendrométricas como o $D A P$, o qual neste estudo apresentou boa correlação com a área de copa.

Relacionando as áreas médias dos indivíduos deste estudo, foi encontrada uma área de copa de $18,05 \mathrm{~m}^{2}$, com desvio padrão de $0,88 \mathrm{~m}^{2}$ e coeficiente médio de variação de 4,63\%, o qual pode ser considerado baixo, principalmente se considerada à alta diversidade de espécies que compõem este estudo. Uma resolução espacial de aproximadamente uma ordem de grandeza menor que o diâmetro médio dos menores indivíduos proporciona bons resultados, levando uma delimitação mais confiável dos limites do dossel (CULVENOR, 2002).

Segundo Erickson (2004), para pesquisa com copas de árvores, a alta resolução espacial significa um pixel correspondendo no máximo a um metro. Nesse sentido, pode-se inferir que o uso do sensoriamento remoto pode ser uma alternativa viável para obtenção de dados que exaustivamente são coletados em nível de campo. Porém, a verdade de campo é essencial para validação destes. Ademais, destaca-se a importância da seleção da melhor resolução espacial e espectral da imagem, ja que possuem influência diretamente no processo de delimitação das copas das árvores.

Este trabalho apresenta uma metodologia alternativa para obter informações como a área de copas de florestas nativas. A classificação supervisionada foi capaz de gerar resultados satisfatórios, os quais exibiram boas relações com dados provenientes da validação de campo. Entretanto, em futuros trabalhos, pretende-se testar o uso de técnicas orientadas a objeto (GEOBIA), que demonstraram resultados mais precisos em outros estudos, bem como a integração de ambas as técnicas.

\section{CONCLUSÕES}

A obtenção do parâmetro área de copa via classificação supervisionada pode servir de base para delimitação de copas. Porém, deve-se ter cautela no processo para não superestimar as áreas, devido à complexidade do dossel de uma floresta nativa.

O uso do sensor Worldview-2 para predição da variável DAP mostrou-se eficaz e com resultados semelhantes aos obtidos em campo. A alta resolução espacial e a presença de oito bandas desse sensor foram importantes para aumentar a exatidão dos resultados.

Para a formação florestal estudada, o uso do sensoriamento remoto, aliado a técnicas de processamento de imagens, gerou resultados satisfatórios para a delimitação de copas de árvores que se destacam no dossel de uma floresta (dominantes). Entretanto, não se deve desconsiderar a realização de uma referência de campo para validar os resultados estimados.

\section{AGRADECIMENTOS}

Ao Conselho Nacional de Desenvolvimento Científico e Tecnológico (CNPq, processo no 305914/2014-6 e $309030 / 2017-0)$ pelo auxílio para a pesquisa e concessão de bolsa.

\section{REFERÊNCIAS}

AMARAL, M. V. F. et al. Avaliação e comparação de métodos de classificação de imagens de satélites para o mapeamento de estádios de sucessão florestal. Árvore, v.3 3, p. 575-582, 2009.

CALLEGARO, R. M. et al. Estrutura do componente arbóreo de uma floresta estacional decidual ripária em Jaguari, RS. Ciência Rural, v. 42, p. 305-311, 2012.

CANAVESI, V. et al. Estimativa de volume de madeira em plantios de Eucalyptus spp. utilizando dados hiperespectrais e dados topográficos. Revista Árvore, v. 34, p. 539-549, 2010.

CANTARELLI, E. B. et al. Diversidade de formigas (Hymenoptera: Formicidae) da serrapilheira em diferentes sistemas de uso do solo. Ciência Florestal, v. 25, p. 605-614, 2015.

CEMETRS - Conselho Estadual de Meteorologia do Rio Grande do Sul. Atlas climático do Rio Grande do Sul. Fundação Estadual de Pesquisa Agropecuária. Disponível em: http://www.r3pb.com.br/ AtlasCemetRS

COSTA, E. A. et al. Influência da posição sociológica na relação hipsométrica de Araucaria angustifolia. Revista Brasileira de Ciências Agrárias - Brazilian Journal of Agricultural Sciences, v. 9, p. 110-116, 2014.

CULVENOR, D. S. TIDA: an algorithm for the delineation of tree crowns in high spatial resolution remotely sensed imagery. Computers \& Geosciences, v. 28, n. 1, p. 33-44, 2002.

DIGITAL GLOBE. Feature classification: worldview-2. Longmont, 2010. Disponível em: http://www.digitalglobe.com

ERIKSON, M. Segmentation and classification of individual tree crowns: in high spatial resolution aerial images. Uppsala: Swedish University of Agricultural Sciences, 2004.

ENVI 5.1. Exelis visual information solutions. Version 5.1. 2014.

FERREIRA, M. P. et al. Mapping tree species in tropical seasonal semi-deciduous forests with hyperspectral and multispectral data. Remote Sensing of Environment, v. 179, p. 66-78, 2016.

FOODY, G. M. Status of land cover classification accuracy assessment. Remote Sensing of Environment, v. 80, n. 1, p. 185201, 2002.

FRANKLIN, S. E. Remote sensing for sustainable forest management. Boca Raton: CRC Press. 2001 
GARSON, G. D. Statnotes: topics in multivariate analysis. 2009. Disponível em: http://faculty.chass.ncsu.edu/garson/PA765/ statnote.html

GOMES, P. B. et al. Segmentação e classificação de dossel florestal em imagens Quickbird. Ambiência, v. 4, p. 35-46, 2008.

JENSEN, J. R. Sensoriamento remoto do ambiente: uma perspectiva em recursos terrestres. São José dos Campos: Parêntese, 2011.

KALAF, R. et al. Landsat8: avanços para mapeamento em mesoescala. $4^{\circ}$ Congresso Brasileiro de Geoprocessamento, 2013.

KOTTEK, M. et al. World map of the Köppen-Geiger climate classification updated. Meteorologische Zeitschrift, v. 15, p. 259263, 2006.

LANDIS, J.; KOCH, G. G. The measurements of agreement for categorical data. Biometrics, v. 33, n. 3, p. 159-179, 1977.

LIMA NETO, E. M. et al. Fotografias aéreas para mensuração da área de copa das árvores de ruas de Curitiba-PR. Floresta, v. 42, p. 577-588, 2012.

MENESES, P. R. Princípios de sensoriamento remoto. In: MENESES, P. R.; ALMEIDA, T. (Eds.). Introdução ao processamento de imagens de sensoriamento remoto. Brasília: CNPq, 2012.

NASCIMENTO, A. R. T. et al. Florística e estrutura da comunidade arbórea de um remanescente de Floresta Estacional Decidual de encosta, Monte Alegre, GO, Brasil. Acta Botanica Brasilica, v. 18, p. 659-669, 2004.

NASCIMENTO, R. F. F. et al. O algoritmo Support Vector Machines (SVM): Avaliação da separação ótima de classes em imagens CCDCBERS-2. 14 Simpósio Brasileiro de Sensoriamento Remoto, p. 2079-2086, 2009.

ODUM, E. P. Fundamentos de ecologia. 6. ed. Fundação Calouste Gulbenkian: Lisboa, 2001.

PONZONI, F. J.; REZENDE, A. C. P. Influência da resolução espacial de imagens orbitais na identificação de elementos da paisagem em Altamira-PA. Revista Árvore, v. 26, n. 4, p. 403-410, 2002.

POULIOT, D. A. et al. Automated tree crown detection and delineation in high-resolution digital camera imagery of coniferous forest regeneration. Remote Sensing of Environment, v. 82, n. 2-3, p. 322-334, 2002.

POWELL, R. et al Sources of error in accuracy assessment of thematic land-cover map in the Brazilian Amazon. Remote Sensing of Environment, v. 90, p. 221-123, 2004.

PUTZ, F. E. The natural history of lianas on Barro Colorado Island, Panama. Ecology, v. 65, p. 1713-1724, 1984.

READ, J. M. Application of merged 1-m and 4-m resolution satellite data to research and management in tropical forests. Journal of Applied Ecology, v. 40, n. 3, p. 592-600, 2003.
SANTOS, M. M. et al. Estimativa de parâmetros florestais em área de cerrado a partir de imagens do sensor Landsat 8. Floresta, v. 47, p. 75-83, 2017.

SANQUETTA, C. R. et al. Estoques de biomassa e carbono em povoamentos de acácia-negra em diferentes idades no Rio Grande do Sul. Scientia Forestalis, v. 12, n. 103, p. 361-370, 2014

SOARES, C. P. B. et al. Dendrometria e inventário florestal. Viçosa: Ed. UFV, 2006. 